\title{
Nanoscale On-Chip Decoupling Capacitors
}

\author{
Mikhail Popovich \\ Qualcomm, Inc. \\ 5775 Morehouse Drive \\ San Diego, California 92121, USA \\ mikhailp@qualcomm.com
}

\begin{abstract}
A distributed on-chip decoupling capacitor network is proposed in this paper to replace one large capacitor. A system of distributed on-chip decoupling capacitors is shown to provide an efficient solution for providing the required on-chip decoupling capacitance under existing technology constraints. In a system of distributed on-chip decoupling capacitors, each capacitor is sized based on the parasitic impedance of the power distribution grid. To be effective, on-chip decoupling capacitors should be charged before the next switching cycle. A design space for estimating the maximum parasitic resistance of the power distribution grid during discharge and the charging phase has been determined. Related simulation results for typical values of the on-chip parasitic resistance are also presented. An analytic solution is shown to provide accurate parameters of the distributed system. The worst case error is $0.002 \%$ during discharge and $0.08 \%$ during the charging phase as compared to SPICE.
\end{abstract}

\section{INTRODUCTION}

Decoupling capacitors are widely used to manage the power supply noise [1]. Decoupling capacitors are an effective way to reduce the impedance of power delivery systems operating at high frequencies [2]. A decoupling capacitor acts as a local reservoir of charge, which is released when the power supply voltage at a particular current load drops below some tolerable level. Since the inductance scales slowly [3], the location of the decoupling capacitors significantly affects the design of the power/ground $(\mathrm{P} / \mathrm{G})$ networks in high performance integrated circuits (ICs) such as microprocessors. At higher frequencies, a distributed system of decoupling capacitors are placed onchip to effectively manage the power supply noise [4].

As described in [5], to be effective, an on-chip decoupling capacitor should be placed such that both the power supply and the current load are located inside the appropriate effective radius. The efficient placement of on-chip decoupling capacitors in nanoscale ICs is the subject of this paper. Unlike the methodology for placing a single on-chip decoupling capacitor presented in [5], a system of distributed on-chip decoupling capacitors is proposed to replace a single decoupling capacitor.

Decoupling capacitors have traditionally been allocated into the white space (those areas not occupied by the circuit elements) available on a die [6]. In this way, decoupling capacitors are often placed at a significant distance from the current load. The efficacy of the distant on-chip decoupling capacitors is greatly reduced. As a result, larger on-chip decoupling capacitors are required. The conventional allocation strategy, therefore, results in increased power noise, compromising the signal integrity of an entire system.

To be effective, a decoupling capacitor should be placed physically close to the current load. This requirement is

\author{
Eby G. Friedman \\ Dept. of Electrical and Computer Engineering \\ University of Rochester \\ Rochester, New York 14627, USA \\ friedman@ece.rochester.edu
}

naturally satisfied in board and package applications, since large capacitors are much smaller than the dimensions of the circuit board (or package) [7]. In this case, a lumped model of the decoupling capacitor provides sufficient accuracy [8].

The size of an on-chip decoupling capacitor, however, is directly proportional to the area occupied by the capacitor and can require a significant portion of the on-chip area. The minimum impedance between an on-chip capacitor and the current load is fundamentally affected by the magnitude (and therefore the area) of the capacitor. Systematically partitioning the decoupling capacitor solves this issue. A system of distributed on-chip decoupling capacitors is illustrated in Fig. 1.

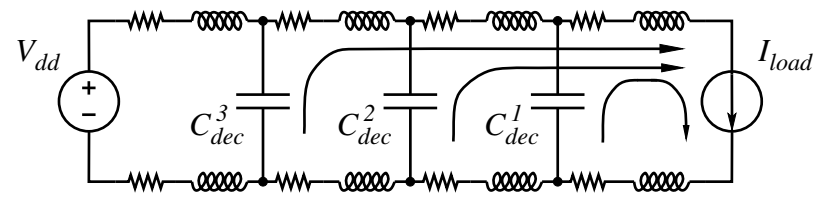

Fig. 1. A network of distributed on-chip decoupling capacitors. The magnitude of the decoupling capacitors is based on the impedance of the interconnect segment connecting a specific capacitor to a current load.

In a system of distributed on-chip decoupling capacitors, each decoupling capacitor is sized based on the impedance of the interconnect segment connecting the capacitor to the current load. A particular capacitor only provides charge to a current load during a short period. The rationale behind the proposed scheme can be explained as follows. The capacitor closest to the current load is engaged immediately after the switching cycle is initiated. Once the first capacitor is depleted, the next capacitor is activated, providing a large portion of the total current drawn by the load. This procedure is repeated until the last capacitor becomes active. Similar to the hierarchical placement of decoupling capacitors presented in [4], the proposed technique provides an efficient solution for providing the required on-chip decoupling capacitance based on specified capacitance density constraints. A system of distributed on-chip decoupling capacitors should therefore be utilized to provide a low impedance, cost effective power delivery network in nanoscale ICs.

The paper is organized as follows. The design of an onchip distributed decoupling capacitor network is presented in Section II. Charging the system of distributed on-chip decoupling capacitors is discussed in Section III. Related simulation results for typical values of the on-chip parasitic resistance 
are discussed in Section IV. Some specific conclusions are summarized in Section V.

\section{DESIGN OF A SySTEM OF DistribUted ON-CHIP DECOUPLING CAPACITORS}

Similar to a chain of tapered buffers, a network of on-chip decoupling capacitors with progressively increasing magnitude is proposed to replace a large on-chip decoupling capacitor, as illustrated in Fig. 2. The magnitude of each decoupling capacitor in the distributed network is determined based on the parasitic resistance of the power/ground lines connecting the decoupling capacitors and the current load. Note that as the capacitor is placed farther from the current load, the magnitude of the on-chip decoupling capacitor is increased due to the larger available area.

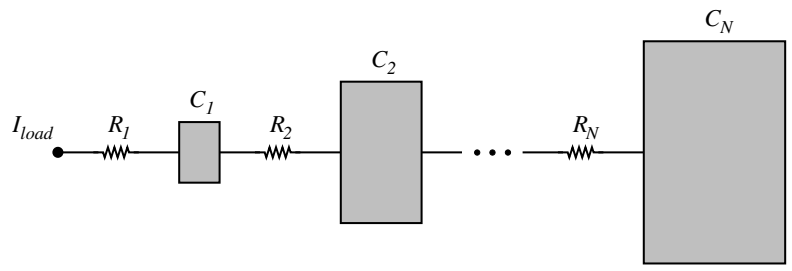

Fig. 2. A physical model of the proposed system of distributed on-chip decoupling capacitors.

A circuit model of the proposed system of distributed onchip decoupling capacitors is shown in Fig. 3. For simplicity, two decoupling capacitors are assumed to provide the required charge drawn by the current load. The impedance of the metal lines connecting the capacitors to the current load is modeled as resistors, $R_{1}$ and $R_{2}$. Note that the on-chip inductance of the power/ground lines is neglected due to the significantly increased complexity of the characterizing system of equations. A system of distributed on-chip decoupling capacitors which includes the parasitic inductance of the metal lines is analytically non-tractable. A triangular current source is assumed to model the current load. The magnitude of the current source increases linearly, reaching the maximum current $I_{\max }$ at rise time $t_{r}$, i.e., $I_{\text {load }}(t)=I_{\max } \frac{t}{t_{r}}$. The maximum tolerable ripple at the current load is $10 \%$ of the power supply voltage.

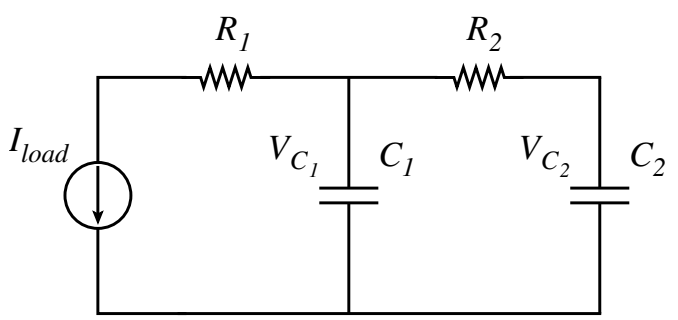

Fig. 3. Circuit model of an on-chip distributed decoupling capacitor network. The impedance of the metal lines is modeled as $R_{1}$ and $R_{2}$, respectively.

The voltage across the decoupling capacitors at the end of the switching cycle $\left(t=t_{r}\right)$ can be determined from
Kirchhoff's laws [9]. Writing KVL and KCL for each of the loops (see Fig. 3), the system of differential equations for the voltage across $C_{1}$ and $C_{2}$ at $t_{r}$ is

$$
\begin{gathered}
\frac{d V_{C_{1}}}{d t}=\frac{V_{C_{2}}-V_{C_{1}}}{R_{2} C_{1}}-\frac{I_{\text {load }}}{C_{1}}, \\
\frac{d V_{C_{2}}}{d t}=\frac{V_{C_{1}}-V_{C_{2}}}{R_{2} C_{2}} .
\end{gathered}
$$

Simultaneously solving (1) and (2) and applying the initial conditions, the voltage across $C_{1}$ and $C_{2}$ at the end of the switching activity can be determined as described in [10].

In general, to determine the parameters of the system of distributed on-chip decoupling capacitors, the following assumptions are made. The parasitic resistance of the metal line(s) connecting capacitor $C_{1}$ to the current load is known. $R_{1}$ is determined by technology constraints (the sheet resistance) and by design constraints (the maximum available metal resources). Note that the voltage across $C_{2}$ after discharge is also a design parameter. Since the capacitor $C_{2}$ is directly connected to the power supply (a shared power rail), the voltage drop across $C_{2}$ appears on the global power line, compromising the signal integrity of the overall system.

The system of equations to determine the parameters of an on-chip distributed decoupling capacitor network as depicted in Fig. 3 is

$$
\begin{aligned}
V_{\text {load }} & =V_{C_{1}}-I_{\max } R_{1}, \\
V_{C_{1}} & =f\left(C_{1}, C_{2}, R_{2}\right), \\
V_{C_{2}} & =f\left(C_{1}, C_{2}, R_{2}\right), \\
\frac{I_{\text {max }} t_{r}}{2} & =C_{1}\left(V_{d d}-V_{C_{1}}\right)+C_{2}\left(V_{d d}-V_{C_{2}}\right),
\end{aligned}
$$

where $V_{C_{1}}$ and $V_{C_{2}}$ are the voltage across $C_{1}$ and $C_{2}$, respectively. Equation (6) states that the total charge drawn by the current load is provided by $C_{1}$ and $C_{2}$. Note that in the general case with the current load determined a priori, the total charge is the integral of $I_{\text {load }}(t)$ from zero to $t_{r}$. Solving (3) for $V_{C_{1}}$ and substituting into (4), $C_{1}, C_{2}$, and $R_{2}$ are determined from (4) to (6).

\section{Charging Distributed ON-CHIP DECOUPLING NETWORK}

Once discharged, the decoupling capacitors must be charged to support the current demands during the next switching event. If the charge on the capacitors is not fully restored during the relaxation time between two consecutive switching events (the charge time), the system of distributed decoupling capacitors will gradually be depleted, becoming ineffective after several clock cycles. A maximum resistance between the power supply and the distributed decoupling capacitor network therefore exists, which is determined during the charging phase for a target charge time. Similar to discharge, the system of distributed on-chip decoupling capacitors should be placed close to the power supply (within the maximum effective radii [5]) to be effective.

A circuit charging a system of distributed on-chip decoupling capacitors is shown in Fig. 4. The impedance of the metal 
lines connecting the capacitors to the power supply is modeled as resistors $R_{2}$ and $R_{3}$. Note that the initial voltage $V_{C_{1}}^{0}$ and $V_{C_{2}}^{0}$ are determined by the voltage drop during discharge. Also note that for a specific charge time $t_{c h}, R_{3}$ is the only design parameter $\left(R_{1}, R_{2}, C_{1}\right.$, and $C_{2}$ are determined from the discharge phase).

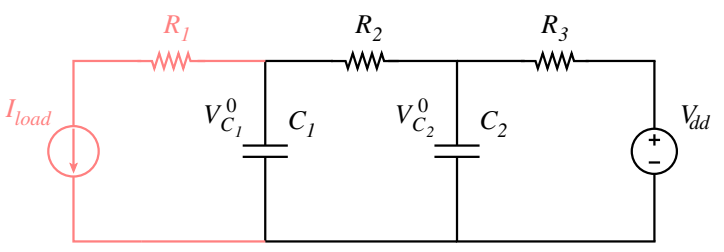

Fig. 4. A circuit charging a system of distributed on-chip decoupling capacitors. The impedance of the metal lines is modeled as $R_{2}$ and $R_{3}$, respectively.

The voltage across the decoupling capacitors during the charging phase can be determined from KVL and KCL for each loop (see Fig. 4). Similar to discharge, the system of differential equations for the voltage across $C_{1}$ and $C_{2}$ at $t_{c h}$ is

$$
\begin{gathered}
\frac{d V_{C_{1}}}{d t}=\frac{V_{C_{2}}-V_{C_{1}}}{R_{2} C_{1}}, \\
\frac{d V_{C_{2}}}{d t}=\frac{V_{d d}-V_{C_{2}}}{R_{3} C_{2}}-\frac{V_{C_{2}}-V_{C_{1}}}{R_{2} C_{2}} .
\end{gathered}
$$

Simultaneously solving (7) and (8) and applying the initial conditions, the voltage across $C_{1}$ and $C_{2}$ at the end of the charging phase can be determined. The maximum resistance $R_{3}$ between $C_{2}$ and the power supply is determined such that both $C_{1}$ and $C_{2}$ are charged to the power supply voltage.

Note that the expressions for determining $R_{3}$ are transcendental. A closed-form solution, therefore, cannot be determined. A design space can be graphically obtained for determining the maximum tolerable resistance $R_{3}$ (or physical distance), permitting the capacitors to be successfully charged by the target charge time. The voltage across $C_{1}$ and $C_{2}$ during the charging phase as a function of $R_{3}$ and $t_{c h}$ is depicted in Fig. 5.

Observe from Fig. 5 that the voltage across $C_{1}$ and $C_{2}$ increases exponentially with the charge time, approaching the power supply voltage for large $t_{c h}$. Note that as $R_{3}$ increases, a larger $t_{c h}$ is required to restore the charge on the decoupling capacitors. Alternatively, the maximum effective frequency of the distributed on-chip decoupling network is reduced. Ideally, the decoupling capacitors should be charge to $V_{d d}$, substantially increasing the required charge time. In practical applications, however, a lower voltage across the decoupling capacitors can be tolerated, significantly increasing the maximum parasitic resistance for a specific $t_{c h}$ (see the black equipotential lines plotted in Fig. 5).

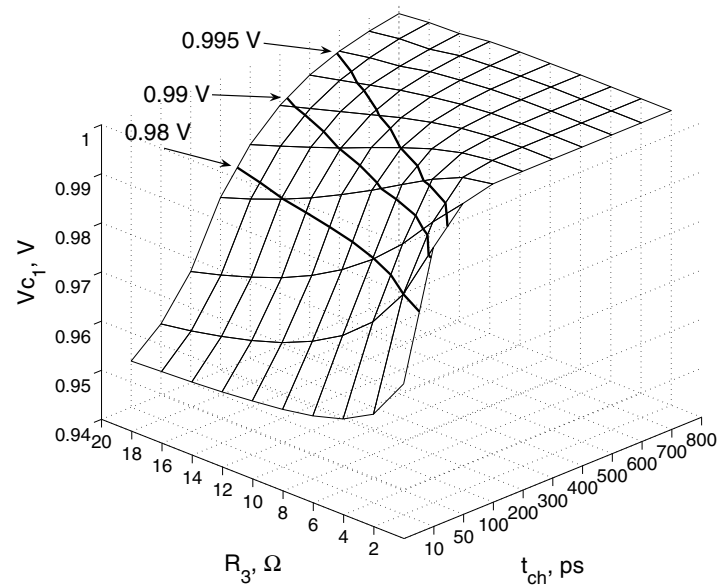

(a)

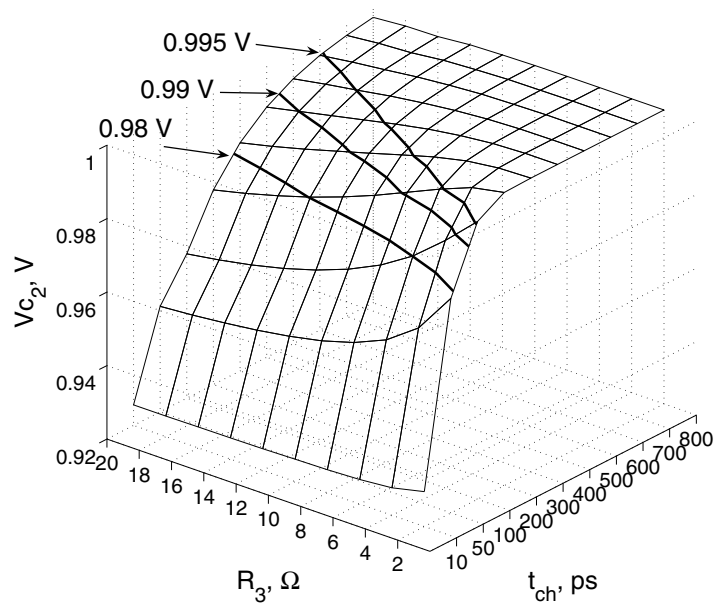

(b)

Fig. 5. Voltage across $C_{1}$ (a) and $C_{2}$ (b) during the charging phase as a function of $R_{3}$ and $t_{c h}: C_{1}=2 \mathrm{pF}, C_{2}=10 \mathrm{pF}, R_{1}=5 \Omega, V_{C_{1}}^{0}=0.91 \mathrm{~V}$, $V_{C_{2}}^{0}=0.95 \mathrm{~V}$, and $V_{d d}=1 \mathrm{~V}$.

\section{Case Study}

The dependence of a system of distributed on-chip decoupling capacitors on the current load and the parasitic impedance of the power delivery system is described in this section to quantitatively illustrate the previously presented concepts. Resistive power and ground lines are assumed and are modeled as resistors. The load is modeled as a ramp current source with a $100 \mathrm{ps}$ rise time. The minimum tolerable voltage across the load terminals is $90 \%$ of the power supply voltage. The magnitude of the on-chip decoupling capacitors for several parasitic resistances of the metal lines connecting the capacitors to the current load during discharge is listed in Table I. The voltage across $C_{1}$ and $C_{2}$ as a function of $R_{3}$ during the charging phase is listed in Table II. Note that the values of $R_{1}, R_{2}$, and $R_{3}$ are typical parasitic resistances of an on-chip power distribution grid for a $90 \mathrm{~nm}$ CMOS technology.

The parameters of the system of distributed on-chip decou- 
TABLE I

THE MAGNITUDE OF ON-CHIP DECOUPLING CAPACITORS AS A FUNCTION OF THE PARASITIC RESISTANCE OF THE POWER/GROUND LINES CONNECTING THE CAPACITORS TO THE CURRENT LOAD

\begin{tabular}{|c|c|c|c|c|c|c|c|c|c|c|}
\hline \multirow{2}{*}{$\begin{array}{l}R_{1} \\
(\Omega)\end{array}$} & \multirow{2}{*}{$\begin{array}{l}R_{2} \\
(\Omega) \\
\end{array}$} & \multirow{2}{*}{$\begin{array}{c}I_{\max } \\
\text { (A) }\end{array}$} & \multirow{2}{*}{$\begin{array}{c}C_{1} \\
(\mathrm{pF})\end{array}$} & \multirow{2}{*}{$\begin{array}{c}C_{2} \\
(\mathrm{pF})\end{array}$} & \multicolumn{2}{|c|}{$V_{\text {load }}(\mathrm{mV})$} & \multirow{2}{*}{$\begin{array}{c}\text { Error } \\
(\%)\end{array}$} & \multicolumn{2}{|c|}{$V_{C_{2}}(\mathrm{mV})$} & \multirow{2}{*}{$\begin{array}{c}\text { Error } \\
(\%)\end{array}$} \\
\hline & & & & & $V_{\text {load }}^{\min }$ & SPICE & & $V_{C_{2}}^{\min }$ & SPICE & \\
\hline 0.5 & 4.5 & 0.01 & 0 & 9.99 & 900 & 89 & 0.0001 & 950 & 949. & 0.0001 \\
\hline 0.5 & 6 & 0.01 & 1.59747 & 6.96215 & 900 & 899.986 & 0.002 & 950 & 949.983 & 0.002 \\
\hline 0.5 & 8 & 0.01 & 2.64645 & 4.97091 & 900 & 899.995 & 0.0006 & 950 & 949.993 & 0.0004 \\
\hline 0.5 & 10 & 0.01 & 3.22455 & 3.87297 & 900 & 899.997 & 0.0003 & 950 & 949.996 & 0.0004 \\
\hline 0.5 & 12 & 0.01 & 3.59188 & 3.17521 & 900 & 899.998 & 0.0002 & 950 & 949.997 & 0.0003 \\
\hline 0.5 & 14 & 0.01 & 3.84641 & 2.69168 & 900 & 899.998 & 0.0002 & 950 & 949.997 & 0.0003 \\
\hline 0.5 & 16 & 0.01 & 4.03337 & 2.33650 & 900 & 899.999 & 0.0001 & 950 & 949.998 & 0.0002 \\
\hline 0.5 & 18 & 0.01 & 4.17658 & 2.06440 & 900 & 899.998 & 0.0002 & 950 & 949.998 & 0.0002 \\
\hline 0.5 & 20 & 0.01 & 4.28984 & 1.84922 & 900 & 899.999 & 0.0001 & 950 & 949.998 & 0.0002 \\
\hline
\end{tabular}

TABLE II

The voltage across $C_{1}$ AND $C_{2}$ As a FunCtion of $R_{3}$ DURing the CHARGING PHASE

\begin{tabular}{|c|c|c|c|c|c|c|}
\hline \multirow{2}{*}{$\begin{array}{l}R_{3} \\
(\Omega)\end{array}$} & \multicolumn{2}{|c|}{$V_{C_{1}}(\mathrm{mV})$} & \multirow{2}{*}{$\begin{array}{l}\text { Error } \\
(\%)\end{array}$} & \multicolumn{2}{|c|}{$V_{C_{2}}(\mathrm{mV})$} & \multirow{2}{*}{$\begin{array}{c}\text { Error } \\
(\%)\end{array}$} \\
\hline & Analytic & SPICE & & Analytic & SPICE & \\
\hline 2 & 999.973 & 999.978 & -0.0005 & 999.957 & 999.965 & -0.0008 \\
\hline 4 & 998.988 & 999.073 & -0.009 & 998.735 & 998.842 & -0.001 \\
\hline 6 & 996.272 & 996.424 & -0.02 & 995.688 & 995.864 & -0.02 \\
\hline 8 & 992.735 & 992.857 & -0.01 & 991.908 & 992.043 & -0.01 \\
\hline 10 & 989.111 & 989.127 & -0.002 & 988.137 & 988.154 & -0.002 \\
\hline 12 & 985.714 & 985.583 & 0.01 & 984.661 & 984.521 & 0.01 \\
\hline 14 & 982.640 & 982.347 & 0.03 & 981.554 & 981.242 & 0.03 \\
\hline 16 & 979.900 & 979.441 & 0.05 & 978.806 & 978.322 & 0.05 \\
\hline 18 & 977.467 & 976.847 & 0.06 & 976.382 & 975.733 & 0.07 \\
\hline 20 & 975.306 & 974.533 & 0.08 & 974.240 & 973.434 & 0.08 \\
\hline \multicolumn{7}{|c|}{$\begin{array}{c}V_{d d}=1 \mathrm{~V}, t_{c h}=200 \mathrm{ps}, R_{2}=5 \Omega \\
C_{1}=2 \mathrm{pF}, \text { and } C_{2}=10 \mathrm{pF}\end{array}$} \\
\hline
\end{tabular}

pling capacitors are analytically determined from (3)-(6). The resulting power supply noise during discharge is estimated using SPICE and compared to the maximum tolerable level (the minimum voltage across the load terminals $V_{\text {load }}^{\text {min }}$ ). The maximum voltage drop across $C_{2}$ at the end of the switching activity is also estimated and compared to $V_{C_{2}}^{\text {min }}$. During the charging phase, the voltage across the distributed decoupling capacitors is analytically determined from (7) and (8) and compared to SPICE. Note that the analytic solution produces an accurate estimate of the on-chip decoupling capacitors for typical parasitic resistances of a power distribution grid. The maximum error in this case study is $0.002 \%$ for discharge and $0.08 \%$ for the charging phase, respectively.

\section{CONCLUSIONS}

A methodology for efficiently placing distributed on-chip decoupling capacitors to replace one large capacitor has been proposed in this paper. A distributed on-chip decoupling capacitor network is an efficient solution for providing the required on-chip decoupling capacitance under existing technology constraints in nanoscale ICs. In a system of distributed on-chip decoupling capacitors, each capacitor is sized based on the parasitic impedance of the power delivery system.
Hierarchically allocating the on-chip decoupling capacitors greatly relaxes the technology constraints for physically distant capacitors, making the distant on-chip decoupling capacitors more effective.

Analytic expressions has been developed for determining the parameters of the system for both the discharge and charging phases. Related simulation results for typical onchip parasitic resistances are also presented, demonstrating the high accuracy of the analytic solution. In the worst case, the maximum error is $0.002 \%$ during discharge and $0.08 \%$ during charging as compared to SPICE.

\section{REFERENCES}

[1] M. Popovich, A. V. Mezhiba, and E. G. Friedman, Power Distribution Networks with On-Chip Decoupling Capacitors, Springer Verlag, 2008.

[2] M. Popovich and E. G. Friedman, "Impedance Characteristics of Decoupling Capacitors in Multi-Power Distribution Systems," Proceedings of the IEEE International Conference on Electronics, Circuits and Systems, pp. 160-163, December 2004.

[3] A. V. Mezhiba and E. G. Friedman, "Scaling Trends of On-Chip Power Distribution Noise," IEEE Transactions on Very Large Scale Integration (VLSI) Systems, Vol. 12, No. 4, pp. 386-394, April 2004.

[4] M. Popovich and E. G. Friedman, "Decoupling Capacitors for MultiVoltage Power Distribution Systems," IEEE Transactions on Very Large Scale Integration (VLSI) Systems, Vol. 14, No. 3, pp. 217-228, March 2006.

[5] M. Popovich, E. G. Friedman, M. Sotman, A. Kolodny, and R. M. Secareanu, "Maximum Effective Distance of On-Chip Decoupling Capacitors in Power Distribution Grids," Proceedings of the ACM Great Lakes Symposium on VLSI, pp. 173-179, April/May 2006.

[6] S. Zhao, K. Roy, and C.-K. Koh, "Decoupling Capacitance Allocation and Its Application to Power-Supply Noise-Aware Floorplanning," IEEE Transactions on Computer-Aided Design of Integrated Circuits and Systems, Vol. 21, No. 1, pp. 81-92, January 2002.

[7] J. Kim, et al., "Separated Role of On-Chip and On-PCB Decoupling Capacitors for Reduction of Radiated Emission on Printed Circuit Board," Proceedings of the IEEE International Symposium on Electromagnetic Compatibility, pp. 531-536, August 2001.

[8] T. Hubing, "Effective Strategies for Choosing and Locating Printed Circuit Board Decoupling Capacitors," Proceedings of the IEEE International Symposium on Electromagnetic Compatibility, pp. 632-637, August 2005.

[9] M. E. Van Valkenburg, Network Analysis, Prentice-Hall, 1974.

[10] M. Popovich, E. G. Friedman, R. M. Secareanu, and O. L. Hartin, "Efficient Placement of Distributed On-Chip Decoupling Capacitors in Nanoscale ICs," Proceedings of the ACM/IEEE International Conference on Computer-Aided Design, pp. 811-816, November 2007. 